\title{
Inhalt.
}

Vorwort zur ersten Auflage

Vorrede zur zweiten Auflage . . . . . . . . . . . . . . . . . . VII

Vorrede zur dritten Auflage . . . . . . . . . . . . . . . . . . . . . . . . IX

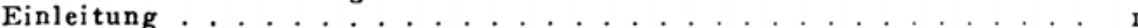

I. Überblick auf die Entwicklung der Hämatologie . . . . . . . . . . . . . I

II. Umfang und Ziele der heutigen Blutforschungen . . . . . . . . . . . . 2

\section{Technik der Blutuntersuohnngen.}

. Die Blutentnahme . . . . . . . . . . . . . . . . . . . 6

2. Die Herstellung angefarbter Prijparate, Nativpriparate . . . . . . . . 8

Beurteilung der Leukocytenzahl und der Menge und Art der Blutzellen . . . . . 8

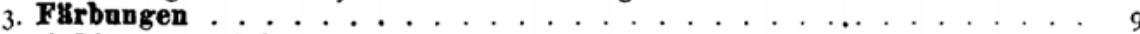

a) Blutausstrichpräparate . . . . . . . . . . . . . 9

Natur der Färbungen. Singuläre, panoptische Färbungen . . . . ... . . ro

Prinzipien der Färbungen. . . . . . . . . . . . . . . . . . . 10

Herstellung gefärbter Präparate . . . . . . . . . . . . . . . . II

Fixationen. . . . . . . . . . . . . . . . 12

Wahl der Färbung . . . . . . . . . . . . . . . . . . . 13

Übersicht über die geeignetsten Färbungen für spezielle Zwecke . . . . . . . 14

Vornahme der Färbungen . . . . . . . . . . . . . . . . 15

Giemsafärbung . . . . . . . . . . . . . . . . . . . I6

- kombinierte . . . . . . . . . . . . . . . . . . 16

— einfache . . . . . . . . . . . . . . . . . . .

Ergebnisse der Färbungen . . . . . . . . . . . . . . . . . . . 16

Modifikationen der Giemsafärbung. . . . . . . . . . . . . . . . . . . . 18

Färbung mit eosinsaurem Methylenblau . . . . . . . . . . . . . . . . . . 18

Jenner-, May-Grünwaldfärbung (Eosinsaures Methylenblau) . . . . . . . . 18

Eosin-Hämatoxylinfärbungen . . . . . . . . . . . . . . . . . 20

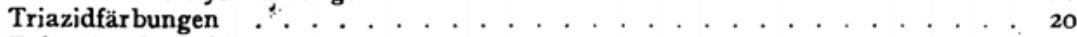

Reine Methylenblaufärbungen . . . . . . . . . . . . . 21

Karbolpyronin-Methylgrünfärbung . . . . . . . . . . . . . . . . . . 22

Dahliafärbung . . . . . . . . . . . . . . . . . . . 22

Methylenblaujodfärbung nach Türk ............ 23

Methoden für die Färbung der Altmann-Schriddeschen Lymphocytengranula

(Mitochondrien, Chondriokonten) . . . . . . . . . . . . 23

Sudanfärbung . . . . . . . . . . . . . . . . . . . 24

b) Färbungen an Organschnitten . . . . . . . . . . . 24

1. Triazidfärbungen nach Sternberg, Fabian . . . . . . . . 25

2. Färbungen mit eosinsaurem Methylenblau nach Zieler, Assmann, Butterfield, Fischer . . . . . . . . . . 25

3. Giemsafärbungen nach Giemsa, Schridde . . . . . . . . . 27

4. Furbungen in der Zahlkammer nach Zollikofer, Riebes, Dunger, Türk 28

5. Vitalfarbangen . . . . . . . . . . . . . . . . . . . . . . . . . . . . . . . . 29

6. Dle ZKhlung der Blutzellen . . . . . . . . . . . . . . . . . . . . . . . . 32

a) Erythrocytenzählung . . . . . . . . . . . . . . . . . . . . 32

b) Die Zählung der Leukocyten . . . . . . . . . . . . . . . . . . . . 37

c) Zählung der Blutplättchen . . . . . . . . . . . . . . . . 40

d) Zählung der Leukocytenarten in gefärbten Trockenpräparaten . . . . . . 4I

7. Die Bestimmung des HHmoglobingehạltes . . . . . . . . . . . . . . . . 43

Hämometer von Sahli . . . . . . . . . . . . . . . . . . . 44

Eichung des Hämometers . . . . . . . . . . . . . . . . . . 46

Das Hämoglobinometer von Sahli-Gowers . . . . . . . . . . . . . . 47

Das Fleischl-Mieschersche Hämometer. . . . . . . . . . . . . 47

Das Kolbenkeilhämometer von Plesch. . . . . . . . . . . . . . . . 47

Das Hämometer von Haldane . . . . . . . . . . . . . . . 47

Das Hämokolorimeter . . . . . . . . . . . . . . . . . . . . . . 47

Das Hämatospektrophotometer . . . . . . . . . . . . . . . . . . $4^{8}$

Die kolorimetrische Doppelpipette . . . . . . . . . . . . . . . . . . . 48

Die Hämoglobinskala von Tallquist . . . . . . . . . . . . . . . . . 49 


\section{Klinische physikalisch-chemische Ontersuchungsmethoden des Blutes.}

Allgemeine Vorbemerkungen .............. Seite

Viskosität des Blutes

Das Viskosimeter von Hess .................. . . $5^{1}$

Die Gewinnung von Serum und Plasma . . . . . . . . . . . . . . . 59

Die Bestimmung des Eiweißes . . . . . . . . . . . . . . . . . . . 60

Andere Methoden zur Ermittlung des Eiweißgehaltes. . . . . . . . . . . . . . 63

Die viskosimetrische Eiweißbestimmung . . . . . . . . . . . . . 63

Die Bestimmung der Albumin- und Globulinprozente im Serum . . . . . . . . . 64

Die Bestimmung des Fibrinogens im Plasma . . . . . . . . . . . . . . 68

Die Bestimmung des spezifischen Gewichtes . . . . . . . . . . . . . . . . . . 69

a) des Gesamtblutes . . . . . . . . . . . . . . . . . . . . 69

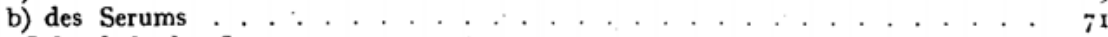

Der Salzgehalt des Serums . . . . . . . . . . . . . . 7 I

Bestimmung des Trockenrückstandes und des Wassergehaltes . . . . . . . 72

Die Bestimmung des Eisens . . . . . . . . . . . . . . . . . . . 73

Die Untersuchung der Serumfarbe. . . . . . . . . . . . . . . . . . 74

Der osmotische Druck des Blutes . . . . . . . . . . . . . . . . 76

Permeabilität und Resistenz der roten Blutkörperchen . . . . . . . . . . . . 77

Technik der Resistenzbestimmung . . . . . . . . . . . . . . . . . 79

Ergebnisse der Resistenzprüfungen . . . . . . . . . . . . . . . . 80

Volumenprozente . . . . . . . . . . . . . . . . . . . $8_{3}$

Technik der Volumenbestimmung . . . . . . . . . . . . . . . . . 86

I. Refraktometrische Bestimmung . . . . . . . . . . . . . . . . . . 86

2. Viskosimetrische Bestimmung . . . . . . . . . . . . . . . . . . 88

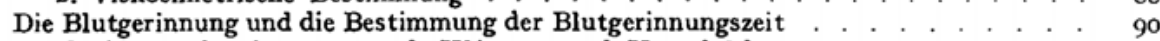

Gerinnungsbestimmung nach Klinger und Herzfeld . . . . . . . . . 96

Ergebnisse der Blutgerinnungsuntersurchung _. . . . . . . . . . . . . . . 97

Alkaleszenzbestimmungen des Blutes . . . . . . . . . . . . . . . 98

Die Bestimmung der Gesamtblutmenge . . . . . . . . . . . . . . . . . . . . . . 99

Sauerstoffzehrung des Blutes . . . . . . . . . . . . . . . . . ${ }^{103}$

Die Oxydasenreaktionen . . . . . . . . . . . . . . . . . . . . 103

I. Die Guajakreaktion . . . . . . . . . . . . . . . . . 103

2. Die Indophenolblausynthese . . . . . . . . . . . . . . . . 104

Die Jodreaktion des Blutes und der Leukocyten . . . . . . . . . . . . . 107

\section{Die roten Blutkörperchen (R.).}

I. Physiologisehe Verhultnisse . . . . . . . . . . . . . . . 112

Allgemeine Verhältnisse . . . . . . . . . . . . . . . . 112

Neuere Ansichten über den Bau der R. . . . . . . . . . . . . . . . . 112

Funktion. Zahl. Färbeindex . . . . . . . . . . . . . . . 116

Untergang . . . . . . . . . . . . . . . . . 117

Physiologische Schwankungen .............. 117

Die Ery.throblasten und die Bildung der R. im postfötalen Leben . . . . . I19

Normoblasten und Megaloblasten . . . . . . . . . . . . . . . . I19

Lymphatische Vorstufen der Erythroblasten . . . . . . . . . . 121

Entkernung . . . . . . . . . . . . . . . . . . 122

Embryonale Blutbildung . . . . . . . . . . . . . . . 123

Vergleichende Anatomie und Embryologie der R.-Bildung . . . . . . . . 126

Ursprung der roten Blutkörperchen . . . . . . . . . . . . . . . . 127

II. 'Pathologisehe Verhultnisse . . . . . . . . . . . . . . . . . . . . . . . . . . . 130

Abnorme Werte der R. in der Raumeinheit . . . . . . . . . . . . . 130

Abnahme des Hämoglobingehaltes . . . . . . . . . . . . . . . . . . . . . I31

Färbeindex . . . . . . . . . . . . . . . . . . ${ }^{132}$

Größen- und Gestaltsveränderungen . . . . . . . . . . . . . . 134

Anisocytose. Makrocyten. Megalocyten. Pessarformen . . . . . . . . . 134

Poikilocytose . . . . . . . . . . . . . . . . . . . 138

Kernhaltige rote Blutkörperchen . . . . . . . . . . . . . . . . . . . . . . ${ }_{138}$

Artefakte und Nekrobiosen . . . . . . . . . . . . . . . . . 140

Veränderungen bei Vitalfärbungen . . . . . . . . . . . . I41

Veränderungen der Tinktionsverhältnisse . . . . . . . . . . . . ${ }_{143}$ 
Polychromasie

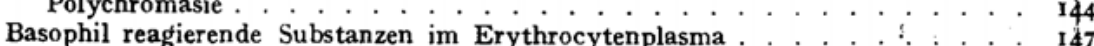

1. Kernbröckel . . ......................... . . . 147

2. Eigenartige Kernabschnürungen an Megaloblasten . . . . . . . . . . . . . 148

3. Howell-Jollykörper . . . . . . . . . . . . . . . . . . . . . . . . . 148

4. Chromatinstäubchen . . . . . . . . . . . . . . . . . . . . I50

5. Ringkörper . . . . . . . . . . . . . . . . . . . . . I5 I

6. Azurophilie (rote basophile) Punktierung bei Giemsafärbung . . . . . . 152

7. Azurophile (rote) Strichelung und Fleckung bei Giemsafärbung . . . . . 153

8. Basophile Punktierung . . . . . . . . . . . . . . . . . . 153

- Vorkommen . . . . . . . . . . . . . . . . . . . . . . . . . 154

- Genese der basophilen Punktierung . . . . . . . . . . . . . . . . 157

-- Nachweis des regenerativen Charakters . . . . . . . . . . . . . . . 160

Pathologisches Wiederauftreten der Erythropoëse in Leber, Milz und Lymphdrüsen 164

\section{Die weiBen Blntkörperchen (Lenkocyten).}

Die Lymphocyten $(\mathcal{L}$.) . . . . . . . . . . . . . . . . . . . . . . . . . . . . 168

Monocyten $=$ große Mononukleäre und Übergangsformen Ehrlichs . . . . . . . . . . 177

Die neutrophilen polymorphkernigen Leukocyten (N.) . . . . . . . . . . . . . . 186

Die eosinophilen Zellen (Eos.) . . . . . . . . . . . . . . . . . . . . . . . . . 189

Die Mastzellen (Ma.) . . . . . . . . . . . . . . . . . . . . . . . . . . . . . . . . . . 200

Pathologisch im Blute auftretende Leukocyten. . . . . . . . . . . . . . . . 205

Myelocyten . . . . . . . . . . . . . . . . . . . . . . . . . . . . . . . . . 206

Myeloblasten (Naegeli) (ungranulierte myeloische Zellen) . . . . . . . . . . . . 209

Pathologische Lymphocyten. . . . . . . . . . . . . . . . . . . . . . . . . . 215

Plasmazellen ............ . . . . . . . . . . . . . . . . . . . . 215

I. Lymphoblastische Plasmazellen. . . . . . . . . . . . . . . . . . . . 216

2. Lymphocytäre Plasmazellen . . . . . . . . . . . . . . . . . . . . . . . 216

3. Radkernplasmazellen . . . . . . . . . . . . . . . . . . . . . . . . . . 216

4. Myeloblastische Plasmazellen . . . . . . . . . . . . . . . . . . . . . . 217

Megakaryocyten. Knochenmarksriesenzellen . . . . . . . . . . . . . . . . . . . 218

Kriterien der Jugend und des Alters der Leukocyten . . . . . . . . . . . . . . 220

Abnormitäten der normalen Blutleukocyten . . . . . . . . . . . . . . . . . . . 22I

(Arnethsche Lehre)

Spezifität der Leukocytenarten . . . . . . . . . . . . . . . . . . . . . . . . 225

Die Bildung der Leukocyten . . . . . . . . . . . . . . . . . . . . . . . . . . . . . . . . . 228

Blut der Embryonen. Embryonale Leukopoëse, bes. nach eig. Forschungen . . . . 230

Resultate der embryonalen Forschung . . . . . . . . . . . . . . . . . . . . . 234

Vergleichende Anatomie und Histologie der Leukocyten und der Leukopoëse . . . 236

Pathologische Leukopoëse der Organe . . . . . . . . . . . . . . . . . . . . . 239

Die vitalen Phänomene und die Funktionen der Leukocyten . . . . . . . . . . . 245

Untergang der Leukocyten . . . . . . . . . . . . . . . . . . . . . . . . 250

\section{Das Knochenmark als Organ.}

Die Leukocytose. . . . . . . . . . . . . . . . . . . . . . . . . 257

Therapeutische Anwendung der Leukocytose . . . . . . . . . . . . 262

Verschiedene Arten der Leukocytose. . . . . . . . . . . . . . . . 263

I. Physiologische Leukoeytosen . . . . . . . . . . . . . . . . . . . . . . . 263

Die Verdauungsleukocytose . . . . . . . . . . . . . . . . . . . . . . . . 263

Die Graviditätsleukocytose . . . . . . . . . . . . . . . . . . . . . . . . . 265

Die Leukocytose der Neugeborenen . . . . . . . . . . . . . . . . . . . . . . . . . . 266

Die Leukocytose nach körperlichen Anstrengungen und thermischen Reizen . . 267

2. Pathologische Leukocytosen . . . . . . . . . . . . . . . . . . . . . . 270

Die Leukocytose bei Infektionskrankheiten . . . . . . . . . . . . . . . . . 271

Die Leukocytose bei Intoxikationen (Toxische Leukocytose) . . . . . . . . . . 273

Die Leukocytose bei Blutungen (poșthämorrhagische Leukocytose) . . . . . . . 273

Die Leukocytose bei malignen Tumoren . . . . . . . . . . . . . . . . . . . 273

Die Leukocytose bei Kachexien . . . . . . . . . . . . . . . . . . . . . . 274

Die Leukocytose der Agone . . . . . . . . . . . . . . . . . . . . . . . . . . . 274

Die Leukocytenschwankungen in der exper. Pathologie und bei Röntgenbestrahlungen 274

Die Leukopenie oder Hypoleukocytose . . . . . . . . . . . . . . . . . . . 277 
Die Lymphknoten and das lymphatlsehe System als Gewrebe

Die Milz als Organ

Histioide Lenkoeyten . . . . . . . . . . . . . . . . . . 287

Die prinziplelle Trennung der lymphatisehen und myeloischen Leukoeyten $29 \mathrm{I}$

Abstammang der Blutzellen . . . . . . . . . . . . . . . . . . 302

Die Abstammung der Blutziellen nach anderen Autoren . . . . . . . . . . 306

Nomenklatur. . . . . . . . . . . . . . . . . . . . . . . 306

\section{Die Blutplättchen.}

Die Blutplättchen . . . . . . . . . . . . . . . . . . . . . 309

Entstehung und Mengenverhältnisse der Blutplättchen. . . . . . . . 31

Die Blutstäubchen . . . . . . . . . . . . . . . 313

Die Anämien.

Allgemeines . . . . . . . . . . . . . . . . . . . . . . . . . . . . . . . . . 316

Vorgetäuschte Anämien . . . . . . . . . . . . . . . . . . . . . . . . . . . 322

Einteilung der Anämien . . . . . . . . . . . . . . . . . . . . . . . . . . . 322

Beziehungen der Anämien zur Leukopoëse . . . . . . . . . . . . . . . . . . . 324

Die posthämorrhagische Anämie . . . . . . . . . . . . . . . . . . . . . . . 325

Experimentelle Anämien . . . . . . . . . . . . . . . . . . . . . . . . . . . 326

Aregenerative (aplastische) Anämien . . . . . . . . . . . . . . . . . . . . . . 328

Dle Chlorose . . . . . . . . . . . . . . . . . . . . . . . . . . . . . . . 331

Vorbemerkungen . . . . . . . . . . . . . . . . . . . . . . . . . . . . . $33 \mathrm{I}$

Entstehung der Chlorose . . . . . . . . . . . . . . . . . . . . . . . . . 333

Frühere Auffassungen der Entstehung der Chlorose. . . . . . . . . . . . . . 336

Symptome . . . . . . . . . . . . . . . . . . . . . . . 337

Klinischer Befund . . . . . . . . . . . . . . . . . . . . . . . . . 338

Formen der Chlorose . . . . . . . . . . . . . . . . . . . . . . . . . . . . . 342

Das Blut bei Chlorose. . . . . . . . . . . . . . . . . . . . . . . . . . . 344

Patholøgische Anatomie . . . . . . . . . . . . . . . . . . . . . . . 350

Diagnose . . . . . . . . . . . . . . . . . . . . . . . . . . . 35I

Differentialdiagnose . . . . . . . . . . . . . . . . . . . . . . . . . . . . 352

Verlauf und Prognose . . . . . . . . . . . . . . . . . . . . . . . . . . . 354

Therapie . . . . . . . . . . . . . . . . . . . . . . . . . . 354

Die pernizlose Antimie . . . . . . . . . . . . . . . . . . . . . . . . . . . 360

Entwicklung der Lehre über Symptomatik und Wesen . . . . . . . . . . . . 360

Ätiologie . . . . . . . . . . . . . . . . . . . . . . . . . . . . . . . 364

A. Anerkannte Ursachen. . . . . . . . . . . . . . . . . . . . . . . . . . 364

B. Zu Unrecht behauptete ätiologische Momente . . . . . . . . . . . . . . 367

Vorkommen . . . . . . . . . . . . . . . . . . . . . . . . . . 371

Symptomatologie . . . . . . . . . . . . . . . . . . . . . . . . . . . . 372

Blutbefunde . . . . . . . . . . . . . . . . . . . . . . . . . . . 378

Verlauf des Leidens . . . . . . . . . . . . . . . . . . . . . . . . . . 384

Diagnose und Differentialdiagnose . . . . . . . . . . . . . . . . . . . . . . . . . 386

Sektionsbefunde. . . . . . . . . . . . . . . . . . . . . . . . . . . . . 39 I

Histologie . . . . . . . . . . . . . . . . . . . . . . . . . . . 392

Wesen der Biermerschen Anämie . . . . . . . . . . . . . . . . . . . . . 394

Genese der perniziösen Anämie .. . . . . . . . . . . . . . . . . . . . . 396

Therapie . . . . . . . . . . . . . . . . . . . . . . . . . . . . . . . 397

Hämolytische Anämien und hämolytischer Ikterus . . . . . . . . . . . . . . . 407

Die Anaemia psendolenkaemica infantum . . . . . . . . . . . . . . . . . 416

Anämien des Kindesalters . . . . . . . . . . . . . . . . . . . . . . . . . . 421

Leukanämie . . . . . . . . . . . . . . . . . . . . . . . . . . . . 425

\section{Die Lenkämien.}

Allgemeines . . . . . . . . . . . . . . . . . . . . 427

Die chronisch myeloische Leukämie. Chronische Myelose . . . . . . . . . . 430

Klinisches Bild ................... . . . . 431

Blutbefunde bei chronisch myeloischer Leukämie. . . . . . . . . . . . . 434

Veränderungen des Blutbildes . . . . . . . . . . . . . . . . . . 436

Atypien des Blutbefundes bei den Myelosen . . . . . . . . . . . . . . 436

Stoffwechsel der myeloischen Leukämie . . . . . . . . . . . . . . . . . 440

Diagnose der chronischen Myelose . . . . . . . . . . . . . . . . . 442 
Pathologische Anatomie.

Histologische Verhältnisse . . . . . . . . 448

Die akute myeloische Leukämie . . . . . . . . . . . . . . . . . . . . . . . . 452

Diagnose der akuten Myelose. . . . . . . . . . . . . . . . . . . . . . . . 457

Myeloisches Chlorom $=$ myeloische Chloroleukämie. . . . . . . . . . . . . . . . 460

Die chronische lymphatische Leukămie . . . . . . . . . . . . . . . . . . . . 465

Klinisches Bild . . . . . . . . . . . . . . . . . . . . . . . . . 465

Verlauf des Leidens (Therapie) . . . . . . . . . . . . . . . . . . . . . . 471

Differentialdiagnose .. . . . . . . . . . . . . . . . . . . . . . . 472

Pathologische Anstomie und Histologie . . . . . . . . . . . . . . . . . . . 473

Die akute lymphatische Leukämie (akute Lymphadenose) . . . . . . . . . . . . 478

Klinisches Bild . . . . . . . . . . . . . . . . . . . . . . . . . . . 479

Klinische Formen der akuten Lymphadenose . . . . . . . . . . . . . . . . 481

Verlauf des Leidens. . . . . . . . . . . . . . . . . . . . . . . . . . . . 484

Differentialdiagnose . . . . . . . . . . . . . . . . . . . . . . . . . . . 484

Pathologische Anatomie und Histologie . . . . . . . . . . . . . . . . . . . 486

Das lymphatische Chlorom = lymphatische Chloroleukämie (Naegeli) . . . . . . 493

Plasmazellenleukämie . . . . . . . . . . . . . . . . . . . . . . . . . . . . . 495

Histiogenese und Wesen der Lymphadenose . . . . . . . . . . . . . . . . . . . 496

Atypische Leukämien und scheinbare Ủbergänge der Leukämien und von Blutkrank-

heiten in Leukämie. . . . . . . . . . . . . . . . . . . . . . . . . . . 497

Histogenese und Wesen der Leukämien . . . . . . . . . . . . . . . . . . . . 500

Die Tumorauffassung der Leukämien. . . . . . . . . . . . . . . . . . . . . . 502

Die Leukämien als Korrelationsstörungen . . . . . . . . . . . . . . . . . . . 506

Leukämie bei Tieren . . . . . . . . . . . . . . . . . . . . . . 508

\section{Der Symptomenkomplex Psendolenkämie.}

Allgemeines . . . . . . . . . . . . . . . . . . 510

Einteilung des klinischen Symptomenkomplexes Pseudoleukämie.

A. Lymphocytome . . . . . . . . . . . . . . . . . . . . . . . . . . . . 511

B. Myelosen . . . . . . . . . . . . . . . . . . . . . . . . . 512

C. Granulome . . . . . . . . . . . . . . . . . . . . . . . . . . 512

Die Lymphosarkomatose . . . . . . . . . . . . . . . . . . . . . . . . . . . . 514

Klinische Formen. . . . . . . . . . . . . . . . . . . . . . . . 515

Das Lymphogranulom . . . . . . . . . . . . . . . . . . . . . . . . . . . . . 518

Formen des Lymphogranuloms . . . . . . . . . . . . . . . . . . . . . . 520

Das tuberkulöse Granulom, Tuberkulöse Pseudoleukämie . . . . . . . . . . . . 527

Das luetische Granulom . . . . . . . . . . . . . . . . . . . . . . . . . . . 529

Die Megalosplenien und die Bantische Krankheit . . . . . . . . . . . . . . . . 529

Splenomegalie Typ Gaucher. . . . . . . . . . . . . . . . . . . . . . . . . . 536

Das Myelom . . . . . . . . . . . . . . . . . . . . . . . . . . . . . . . . 538

Die Krankheit Polyglobulie . . . . . . . . . . . . . . . . . . . . . . . . . . . 543

Polyglobulie unter anderen Verhältnissen. . . . . . . . . . . . . . . . . . . . . 550

Polyglobulie im Höhenklima . . . . . . . . . . . . . . . . . . . . . . . . . 553

Erklärung der Höhenpolyglobulie . . . . . . . . . . . . . . . . . . . . . . . . 555

Hämorrhagische Diathesen . . . . . . . . . . . . . . . . . . . . . . . . . 558

Skorbut . . . . . . . . . . . . . . . . . . . . . . . . . . . . . . . . . 560

Hamophilie . . . . . . . . . . . . . . . . . . . . . . . . . . . . . . . 560

\section{Infektionskrankheiten.}

Allgemeines . . . . . . . . . . . . . . . . . . . . . . . . . . . . . . . . . . . . . 564

Kruppöse Pneumonie . . . . . . . . . . . . . . . . . . . . . . . . . . . . . 566

Typhus abdominalis . . . . . . . . . . . . . . . . . . . . . . . . . 568

Abnorme und komplizierte Formen . . . . . . . . . . . . . . . . . . . . . $57^{2}$

Diagnostischer Wert der Leukocytenuntersuchung bei Typhus . . . . . . . . . 572

Typhus exanthematicus . . . . . . . . . . . . . . . . . . . . . . . . . 577

Febris wolhynica. . . . . . . . . . . . . . . . . . . . . . . . . . . . 578

Icterus infectiosus . . . . . . . . . . . . . . . . . . . . . . . . . . . . 578

Dysenterie. . . . . . . . . . . . . . . . . . . . . . . . . . . . . . . 579

Pappatacifieber . . . . . . . . . . . . . . . . . . . . . 579 
Diphtherie

Scarlatina

Morbilli.

Rubeolae

Erythema infectiosum . . . . . . . . . . . . . . . . . . . . . . . . . . . . . . . 585

Erysipelas . . . . . . . . . . . . . . . . . . . . . . . . . . . . . . . 585

Variola . . . . . . . . . . . . . . . . . . . . . . . . . . 586

Varicellen . . . . . . . . . . . . . . . . . . . . . . . . . . . . . . . . . . 588

Influenza . . . . . . . . . . . . . . . . . . . . . . . . . . . . . . . . . . 588

Grippe . . . . . . . . . . . . . . . . . . . . . . . . . . . . . . . . . . 589

Tetanus . . . . . . . . . . . . . . . . . . . . . . . . . . 590

Parotitis epidemica . . . . . . . . . . . . . . . . . . . . . . . . . . . . 590

Lyssa . . . . . . . . . . . . . . . . . . . . . . . . . . . . . . . . . . $59 \mathrm{I}$

Anthrax . . . . . . . . . . . . . . . . . ......... . . 591

Actinomycosis . . . . . . . . . . . . . . . . . . . . . . . . . . . . . . . . 591

Cholera . . . . . . . . . . . . . . . . . . . . . . . . . 591

Maltafieber . . . . . . . . . . . . . . . . . . . . . . . . . . . . 592

Dengue . . . . . . . . . . . . . . . . . . . . . . . . . . . . 592

Leishmaniosis . . . . . . . . . . . . . . . . . . . . . . . . . . . . . . . . 592

Trypanosomiasis . . . . . . . . . . . . . . . . . . . . . . . . . . . . . 593

Febris recurrens. . . . . . . . . . . . . . . . . . . . . . . . . . . 593

Polyarthritis acuta. . . . . . . . . . . . . . . . . . . . . . . . . . 593

Sepsis. . . . . . . . . . . . . . . . . . . . . . . . . . . . . . . . . . . . 594

Eiterungen . . . . . . . . . . . . . . . . . . . . . . . . . . . 595

Gynäkologische Affektionen mit Eiterungen. . . . . . . . . . . . . . . . . . . 599

LeberabszeB . . . . . . . . . . . . . . . . . . . . . . . . . . . . 600

Eiterige Meningitis und Genickstarre. . . . . . . . . . . . . . . . . . . . . . 601

Tuberkulose . . . . . . . . . . . . . . . . . . . . . 602

Tuberkulininjektionen . . . . . . . . . . . . . . . . . . . . . . . . . 603

Lymphknotentuberkulose . . . . . . . . . . . . . . . . . . . . . . . . . 604

Lepra . . . . . . . . . . . . . . . . . . . . . . . . . . . . . . . . . . . 607

Syphilis . . . . . . . . . . . . . . . . . . . . . . . . . . . 607

Pertussis . . . . . . . . . . . . . . . . . 610

Malaria .. . . . . . . . . . . . . . . . . . . . . . 610

Helminthiasis.

Ankylostomum duodenale und Necator americanus . . . . . . . . . . . . . . . 614

Botriocephalus latus . . . . . . . . . . . . . . . . . . . . . . . . 616

Tänien . . . . . . . . . . . . . . . . . . . . . . 617

Trichocephalus dispar . . . . . . . . . . . . . . . . . . . . . . . . . . . . 617

Ascaris lumbricoides und Oxyuris vermicularis . . . . . . . . . . . . . . . . 618

Anguillula stercoralis und intestinalis. . . . . . . . . . . . . . . . . . 618

Distomum haematobium. Bilharzia . . . . . . . . . . . . . . . . . 618

Filaria sanguinis... . . . . . . . . . . . . . . . . . . . . . 619

Trichinosis . . . . . . . . . . . . . . . . . . . . . . . 619

Echinokokkus . . . . . . . . . . . . . . . . . . . . . . . 62I

Maligne Tumoren.

Maligne Tumoren . . . . . . . . . . . . . . . . . . . . 622

Vergiftungen und Blutgifte . . . . . . . 628

Globulicide Gifte . . . . . . . . . . . . . . . . . . . . . . . . . . . . . 629

Bleivergiftung . . . . . . . . . . . . . . . . . . . . . . . . . . . . 629

Intoxikationen, die das Hämoglobin chemisch verändern . . . . . . . . . . . 633

Gifte, die das Hämoglobin in Methämoglobin verwandeln . . . . . . . . . . 633

Hämolytische Gifte . . . . . . . . . . . . . . . . . . . . . . . . . . . 634

Paroxysmelle Hämoglobinuric . . . . . . . . . . . . . . . . . . . . . . . . . 635

Erkraukungen der Organe mit innerer Sekretion . . . . . . . . . . . . . . . . . 639

Affektionen des vegetativen Nervensystems . . . . . . . . . . . . . . . . . . 640

Sachregister ................... . . . 648 